\title{
Nurses' perceptions and practices regarding oral care in hospitalized patients: a qualitative study on missed nursing care
}

Melanie Charalambous ( $\square$ melia.charalambous@gmail.com )

Kypriake Demokratia Ypourgeio Ygeias

\section{Georgios Efstathiou}

Kypriake Demokratia Ypourgeio Ygeias

Persofoni Lambrou-Christodoulou

Kypriake Demokratia Ypourgeio Ygeias

Iraklis Irakleous

Kypriake Demokratia Ypourgeio Ygeias

Evridiki Papastavrou

Cyprus University of Technology

Research article

Keywords: Nurses' perceptions, nurses' practices, nurses' knowledge, oral care, hospitalized patients

Posted Date: September 3rd, 2020

DOl: https://doi.org/10.21203/rs.3.rs-41564/v1

License: (c) (i) This work is licensed under a Creative Commons Attribution 4.0 International License. Read Full License 
Nurses' perceptions and practices regarding oral care in hospitalized patients: a qualitative study on missed nursing care

Melanie Charalambous, PhD, RN Educational Sector, Nursing Services, Ministry of Health, Cyprus

Georgios Efstathiou, PhD, RN Educational Sector, Nursing Services, Ministry of Health, Cyprus

Persefoni Lambrou-Christodoulou, PhD, Dentist

Iraklis Irakleous, MSc, RN Educational Sector, Nursing Services, Ministry of Health, Cyprus

Evridiki Papastavrou, PhD, RN Associate Professor of Nursing, Department of Nursing, Cyprus University of Technology, MC Chair COST Action 15208 RANCARE

Corresponding author: Melanie Charalambous 


\begin{abstract}
BACKGROUND: Oral health is considered essential for individuals' well-being. Poor oral health has a negative impact on overall human health while contributing and predisposing to other diseases. It has been internationally documented that nurses delay or omit oral care of hospitalized patients and as a result of this, the risk of adverse events is increased.
\end{abstract}

METHODS: Four focus groups were organized $(n=30)$ to elicit the issue under study. Nurse managers and nurses working in medical and surgical departments in 3 acute care hospitals participated in June 2019. The discussions were coordinated by an independent moderator, using a semi-structured interview guide. Recordings of the conversations were transcribed and thematic analysis was performed according to Krueger and Casey guidelines.

RESULTS The findings confirm that oral care of hospitalized patients is often omitted and is not acknowledged as an important patient need. The barriers and facilitators that affected oral health care positively or negatively were divided into three themes and included patient' characteristics, nurses' knowledge and skills and recourses as well as the working environment.

CONCLUSIONS: Oral care is not considered as priority and there are many factors that contribute negatively or positively to its implementation. Nurse managers and nurses can play a crucial role for the adoption of universal oral care practices in hospitalized patients.

Key words: Nurses' perceptions, nurses' practices, nurses' knowledge, oral care, hospitalized patients 


\section{BACKGROUND}

Oral disease remains a major public health burden worldwide and it is one of the most common public health issues with significant socio-economic impact [1]. Oral disease and non-communicable diseases are closely interlinked through sharing common risk factors and underlying infection/inflammatory pathways. It is of great importance to integrate oral health into global health agenda via the common risk factor approach. The long-term sustainable strategy for global oral health should focus on health promotion and disease prevention through effective multidisciplinary teamwork [2].

Several systemic diseases have oral manifestations and have different effects on general health. Improved oral hygiene reduces the progression or occurrence of periodontal disease, characterized by the destruction of the supporting tissues of the tooth [3] and it prevents respiratory disease among high-risk elderly adults living in nursing homes and patients in intensive care units. Studies support the significance of oral and dental factors while controlling for established medical risk factors in chronic bronchitis, pneumonia, and chronic pulmonary obstructive disease $[4,5,6]$. Furthermore, many oral/periodontal pathogens have been reported to predispose to cardiovascular diseases [7]. Tooth loss resulting from periodontal disease is related to subclinical atherosclerosis and can increase the death risk and death from upper gastrointestinal cancer, heart disease and stroke events [8]. Furthermore, poor oral health is related to a higher risk of developing hepatocellular carcinoma and the presence of oral bacteria in cystic pancreatic tumors is associated with the severity of the tumor [9]. Having in mind the poor oral care and its consequences in hospitalized patients, especially the elderly who usually suffer from multimorbidities and sometimes 
are immunosuppressed, it is recognized that the high quality and systematic oral care by the nurses is of great importance.

Oral health is considered as a mirror through which the general health is reflected [10] and it has significant impact on people's well-being and quality of life. However, the circumstances surrounding hospitalisation and ill-health can lead to omitting of oral hygiene. Most of the studies that explored missed nursing care reported that mouth care is a low priority nursing procedure, is very often left behind undone and it is not considered as a fundamental patient need $[11,12]$. Various studies $[13,14,15,16,17]$ support that unfinished nursing care is a highly prevalent problem with negative consequences for patients and health care professionals and there are several reasons leading to it. These include labour resources (85\%), material resources (56\%), and communication (38\%). In the face of scarcity, nurses may inevitably delay or omit interventions like oral health hygiene and give priority to other medical interventions [18].

Unfinished nursing care increases the risk of adverse patient outcomes and threatens safety, quality, and dignity in care [19]. Care of the mouth is an important aspect of care that needs to be carried out consistently. Nurses play a vital role in providing effective oral care and promoting oral hygiene [20].

To our knowledge there are not any studies on nurses' view regarding oral health. Therefore, the main aim of this study was to explore nurses' perceptions and first line nurse managers on the importance of oral health of hospitalized patients and investigate their views, practices and experiences. It is expected that this study will increase knowledge that may allow for increasing acknowledgment of the problem and minimize missed oral care. 
The following research questions were set:

- What is the experience of nurses about oral care in hospitalised patients?

- What are the perceptions of nurses regarding the importance of oral care in hospitalized patients?

- What is the opinion of the participants about the factors that influence positively and negatively the oral care provided to hospitalized patients?

\section{METHODOLOGY}

\section{Design - Data Collection}

The study employed a qualitative research design, with the use of four focus groups. The main reason is the fact that missed nursing care is considered a sensitive issue [21] which may raise feelings of possible self-defense. Therefore, focus groups would give the opportunity to nurses to express their opinions and feelings in more safe and non-blaming environment within the group. A purposive sample of 30 nurses participated in this study. All nurses worked in departments in which patients required assistance regarding their oral care. It was anticipated that these nurses would provide sufficient information as they had daily contact with oral health care. All were practicing nurses from different hospital settings such as surgical, medical and intensive care units. Eight nurses participated in each one of the three focus groups and the fourth one consisted of six nurse first line managers. Of the participants, 24 were female and 6 were male nurses with a mean age 37 years and mean experience at 15 years. The inclusion criteria were willingness to participate and over two years of working experience in hospital environment. Each group was homogenous in term of nurses' background (clinical nurses who provide direct care to hospitalized patients) and heterogenous in terms of age and experience. 
All of the discussions were undertaken in a quiet environment out of the hospital. The discussions were coordinated by a facilitator who had no relation to the participants, using a semi-structured interview guide. The research team developed the content of the preliminary version of the guide and structured the final version. The mean duration of the discussion was 60 minutes. An observer was also present in order to record the non-verbal communication signs. The focus group took place in June 2019. Prior to the study, all participants had received a short document describing the phenomenon of missed care, especially within the oral care context.

\section{ETHICAL CONSIDERATIONS}

This study was reviewed and approved by the National Bioethics Committee. Nurses' participation was voluntary and the purpose and methodology of the study were explained both verbally and written, to the participants. Anonymity and confidentiality were maintaining throughout the study. Patients were informed that all data collected would be destroyed following the completion of data analysis and interpretation. The ethical principles of the World Medical Association Declaration of Helsinki were applied throughout the study.

\section{DATA ANALYSIS}

Data analysis was performed independently by three investigators (MC, GE and PL) following the Krueger and Casey guidelines [22]. Data were transcribed verbatim and thematic analysis was performed to analyze data in order to provide a systematic, ruleguided technique for interpreting the informational contents. More specifically, investigators followed all steps of the analysis. The transcripts of all groups were read several times before starting the process of analysis. This included locating and coding words and phrases relevant to dignity of patients. The themes that emerged were 
compared between the transcripts for relevance and discrepancies which were

\begin{tabular}{|c|c|}
\hline THEMES & SUBTHEMES \\
\hline \multirow[t]{3}{*}{ Importance of oral care in hospitalized patients } & The Positive effect on patients' health \\
\hline & $\begin{array}{l}\text { The negative effects of neglect and of lack of } \\
\text { prevention }\end{array}$ \\
\hline & Burden on health systems \\
\hline \multirow[t]{3}{*}{ Application of oral care } & Not applied \\
\hline & Partial application \\
\hline & Application \\
\hline \multirow[t]{3}{*}{ Barriers and facilitators of oral care } & Patients' characteristics \\
\hline & Nurses' Knowledge, attitude, skills \\
\hline & Working environment \\
\hline
\end{tabular}

resolved by the investigators accordingly. Within each research question several themes emerged (table 1).

Table 1 Themes and Subthemes of the analysis

\section{RESULTS}

1. Nurses perceptions about the importance of oral care in hospitalised patients

\subsection{The Positive effect on patients' health}


Most of the nurses suggested that oral care has a positive effect on hospitalized patients. One nurse said: "You feel jocund when your mouth is clear .... the patients tell us afterwards: now I am relieved, now I feel like a human, now I feel better..." oral hygiene helps to increase the patient's confidence" said another nurse

and

“.... it helps the patient's psychology when he has a clean mouth..." argued one female young nurse

\subsection{The negative effects of neglect and of lack of prevention}

Throughout the discussions, nurses suggested that some negative effects arise when they neglect patients' oral care. One nurse said: "Oral hygiene causes very unpleasant conditions or fungi...."

"It is associated with other more serious diseases and it may affect other organs... even endocarditis" another male nurse said

The patients' medical history and the reason of their admission to hospital, are of great importance in relation to the oral care neglect. A nurse working in ICU reported: "For people who are critically ill, neglecting oral hygiene may also cause some illnesses such as respiratory infection"

while another oncology nurse said: "oral hygiene is very important for the immunosuppressed patients ... when they have oral mucositis, they may present oral cavity's bleeding which it is a very serious side effect for such population..."

\subsection{Burden on health systems}


Burden on health systems was described as a very important reason for providing a factor that is very important for oral care in hospitalized patients.

A nurse stated: "The neglect of oral care and the side effects that will occur, will certainly increase the cost of hospitalization... when the length of hospitalization increases, the costs will increase too"

Further words of a male nurse:

"... and our work is burdened... due to complications deriving from poor oral hygiene, the patient stays longer in hospital, the in-hospital infections increase, and of course our work... this is because we don't pay much attention on oral hygiene and the consequences from its neglect ..."

\section{Application of oral care from nurses}

It was found that in most of the nursing departments, oral cavity care is largely neglected and nurses recognize that they missed it. Particular emphasis on oral care is given in oncology departments as well as in Intensive Care Units compared to medical and surgical departments.

A nurse suggested that oral hygiene is not one of the interventions that staff has in priority during the daily practice:

"I think oral hygiene is pretty much neglected.... we do not pay too much emphasis as we consider other processes more important...."

Most of the nurses agreed that patients' characteristics are of great importance: 
"We neglect them enough .... Mobile patients can take care of themselves and of their mouths, but patients who are bedridden, do not always receive adequate care" a male nurse reported

And

"In the department I work, oral hygiene is thoroughly done due to the immunosuppression of patients but from my previous experience in some other general wards, the percentage of oral care omission was very high..."

A junior female nurse said: "I remember when I was a student in a medical department, I used to take care of the oral cavity every day with my teacher while the staff didn't use to take care of the oral cavity at all ... today we confront the same situation most of the time..."

In addition, nurse managers reported that their staff encourage mobile patients to perform their own oral care. In patients who are bedrest, oral care is applied in some cases from nurses, especially in critically ill and immunosuppressed patients. They mentioned that sometimes oral care cannot be carried out in the right way because of the patients' general condition.

One nurse manager argued:

"In my ward, it is a priority.... we are dealing with patients with leukemia, so when a patient is for therapy, one of the first things we explain to him is the importance of oral care and that is required at least three times a day."

“.... the care of our patients depends entirely on us ...there is a written guideline in the monitoring system reminding staff that every 6 hours there should be done oral care, with the use of an antiseptic oral solution and specific sponges" 
a nurse manager in intensive care unit said.

\section{The barriers and facilitators of oral care}

The barriers and facilitators that affect oral health care positively or negatively were divided into three themes: patient (status, attitudes and willingness), nurse (knowledge, attitudes and skills) and resources and working environment.

\subsection{Barriers of oral care}

\subsubsection{Patients}

One of the main barriers that nurses reported was the patients' general condition. According to the results of this research, the general patients' condition sometimes allows consistency in oral care and sometimes not. For example the severe condition of a patient such as cancer patients or intubated patients allows frequent care while the good condition of a surgical patient leads to neglected oral care. One participant said:

"The severity of the patient is very important ... if my patient is in danger and I have to act immediately, I will not pay attention to the oral cavity ..."

It was also stated:

"It does not immediately threaten the patient life, even though it can cause long term problems ... we leave it behind".

Another barrier that emerged through the present study was the patients' desire but also the effective cooperation between nurse and them. Patients sometimes refuse to 
accept either active or passive care following the given instructions. A nurse mentioned:

"But also, the patient himself ... if he does not cooperate, we cannot perform oral hygiene ..."

Another male nurse support:

“...Well, the patient may not want it himself...we cannot force him to accept oral hygiene”

Another factor that was found to be an important barrier for systematic oral care in hospitalized patients is the mental illness:

"Psychiatric patients have many issues with oral care .... this population cannot fully comply or co-operate effectively"

a young nurse said.

\subsubsection{Nurses}

The present study shows that nurses' attitudes and skills play an important role in the implementation of oral hygiene. It appears that up to date knowledge of nurses is crucial for systematic patients' oral care. Some words from nurses which support the findings:

.."Our culture doesn't allow us to point out proper oral hygiene...» a female nurse said

.."it doesn't look so dirty ... for example we will choose to change the sheets because they look so dirty» another young nurse mentioned

and

"the nurses are bored to deal with it ... some of them feel disgusted with this intervention ..." "' 
A male senior nurse reported that: "There is a lack of knowledge about the consequences of oral care neglect..."

\subsubsection{Working environment}

Nurses supported that workload, nurse managers, attitudes and guidelines are the most common reasons that lead nurses not to take care of patients' oral cavity.

One nurse commented:

"... We cannot take care of the patient properly... it is a matter of time... it is a matter of priorities because some other jobs have to be completed...."

Another nurse who supported the above said:

"Workload is the first factor that hinder us from applying oral care..."

Except of the working environment, work documentation is another one of the major barriers that nurses believe that is a good reason of non-compliance. A surgical nurse mentioned:

"You do not need to document that this is being done.... When there isn't any support from the nurse manager that should be done, it is neglected..."

Furthermore a nurse argued: .... "There are no guidelines on how to take care of the oral cavity..."

\subsection{Facilitators of oral care}

Nurses involved in the current research emphasized that there are important factors that could positively contribute to the systematic care of oral cavity in hospitalized patients. The main issues that were arisen concern again nurses, patients and working environment. 


\subsubsection{Patients}

An important facilitator of oral care that nurses suggested is the role of patients during their stay in hospital. Most of them believe that there is a need to raise awareness between themselves and their relatives about oral hygiene and its importance. Some suggestions derived from their words:

"We must encourage the individual to become aware..."

"It will be very helpful our involvement in educating people about oral hygiene..."

“..By stimulating and engaging the family in oral hygiene."

\subsubsection{Nurses}

Nurses play a crucial role on oral care of hospitalized patients and this was confirmed by all the nurses who took part in the four focus groups. Very interesting were the words of a nurse with many years of clinical experience:

"I only want to say three words: conscientiousness, enough time and holistic nursing care...."

Suggestions on standard procedures so that interventions like oral hygiene not to be forgotten has been a big part of the discussions between the groups. It was stated:

"It is good to have a protocol for oral hygiene and be signed by the nurse every time he/she follows it.."

And

"If we had something like check list, it would be very helpful during our daily routine.." 
Another topic that was much discussed was the development of seminars and specific oral hygiene training. This, could improve nurses' knowledge but also make them aware on the importance of oral care.

"Our organizations have to carry out lectures in relation to the importance of oral hygiene both to health care professionals and to public..." a young female nurse said. Furthermore one other male nurse said: "We need to attend some seminars....it will help us to remember the right procedures in the context of professional development..."

\subsubsection{Working environment}

The role of the nurse manager was described to be crucial to the universal implementation of oral hygiene. Nurses' suggestions focus on nurses' managers frequent monitoring of nurses and other caregivers during the daily practice.

Below are the words of a male nurse:

"The nurse managers have to incorporate oral care into our daily practice. If they decide and insist on its implementation, then we will try to apply oral care..."

Another nurse stated:

"Nurse managers are the key persons. They have to supervise staff frequently. If they persist, then we will start to apply oral care more systematically"

The availability of equipment/consumables was found to act positively to an effective oral care for hospitalized patients. It emerged from discussions that equipment and supplies are often a deterrent to proper oral care. Nurses recommended that practices should be upgraded and relevant materials should be always available.

A nurse who works in a medical department said: 
"Sometimes we don't have enough consumables. Staff who is responsible for this, must order supplies promptly, otherwise we can't work effectively..."

Both oral and written information and education about oral care, to patients, nurses and family was the third most important suggestion according to the nurses' views who participated in this study.

"Oral care must be performed based on the latest guidelines and of course we have to participate in short courses in order to refresh our knowledge...." was pointed out by a young male nurse.

One said:

"By publishing booklets regarding oral care for each population.... this will help raise awareness..."

Another suggested:

"Printed instructions to patients on admission and discharge ...in this way patients will remember it and will be informed".

On the other hand, nurse managers agreed that the implementation of oral hygiene depends on the number of hospitalized patients and the workload, the number of nurses on duty and the priorities set during a shift. They also confirmed that there are relevant guidelines on oral care and they report that their supervision will help nurses not to omit oral care of their patients.

Some words from a nurse manager:

"We take care of oral cavity when we have patients with a very poor general condition, who obviously need it. But we can't apply oral care for all patients ... it is impossible 
because we usually are understaffed and we have to set some priorities because of the workload..."

«We set priorities anyway, in nursing we always set priorities and unfortunately some things are left behind .... one of them is oral care, to be honest...»

What a nurse manager said about supervision:

"Our frequent supervision helps a lot in all processes. If nurses know that you will not check them, they do nothing..."

\section{DISCUSSION}

To our knowledge this is the first research study conducted to investigate nurses' perceptions and practices regarding oral care in hospitalized patients. Oral care of hospitalized patients is one of the most important principles of nursing care aiming to maintain and promote comfort, health and consequently the general quality of life. The impact on outcomes of missed nursing care, such as oral hygiene, has been demonstrated earlier by research $[23,24]$. Promoting oral health of hospitalized

patients with the use of evidence-based practice is of great importance. The present study showed that the majority of the nurses and nurse managers agreed that oral care is very important for patients' nursing care during their stay in the hospital environment even if not implemented.

\section{Nurses perceptions about oral care}

The findings of this study demonstrate that oral care for patients is not a priority for the nursing staff. It was strongly supported that such practices should become a routine part of daily nursing duties. Unfortunately, it was reported that the oral care 
is undertaken only in units with highly recommended nursing care such as Oncology and ICU departments. These findings agree with current literature [25, 26,27,28,29]. Participants reported different barriers and facilitators that play important role in the oral care implementation. Literature supports that, when nurses cannot provide the care they feel is necessary for their patients, they report dissatisfaction with their work, thus leading to distress and work turnover [28].

\section{Barriers of oral care}

The majority of the nurses and nurse managers noted that there are some very important barriers regarding oral care in hospitalized patients. These include the patient characteristics, nurses' knowledge, attitudes and skills and working environment.

Firstly, patients may belong to specific group of patients such as critically ill ventilated patients, patients with cancer, people with mental illness and older people $[27,29,30]$. This leads to the conclusion that nurses discriminate - based on severity, chronicity or type of disease which means that they may violate the principles of the code of ethics [19].

Secondly, when nurses are trained and supported, they respond positively and participate actively in the oral care of their patients $[31,32,33]$. On the other hand, based on results of the current study, the impact of oral hygiene on a person's wellbeing and health status is less recognized by nurses and this is supported by existing literature [34]. In this context, inadequate knowledge has the potential to undermine the quality of patient care [35].

Thirdly and in relation to the working environment, nurses reported that workload was one of the major factors that influence oral care. They supported that in workload cases they decide to perform priorities of the nursing interventions. Oral care is not consider 
as a main priority. These findings are strongly supported from the current literature [19]. In a recent study that was conducted by Pai et al. (2019) investigating and asking about the barriers of oral care, nurses reported that lots of writing tasks, low staffing, and different practices were the major barriers to oral care [36]. Furthermore, the variety of hospital provided supplies and the availability of them are major factors that were found to affect oral hygiene in inpatients and equipment for oral patient care can greatly affect the quality of care given by the nurses [37]. Sometimes, the absence or necessary toothbrushes hindered nurses from providing oral care during their daily routine. Approximately more than half nurses who participated in the current research agreed that they don't use the proper ideal consumables for their patients.

\section{Facilitators of oral care}

Facilitators that enhanced oral care, according to nurses' opinions, are divided into three categories and these are patient and nurses-centered and system focused (working environment, nurse managers).

Nurses supported that patients and relatives should be informed about the importance of oral hygiene and getting involved on it, in various ways.

In addition, nurse's role is crucial in order to facilitate oral care in their daily routine and they have to implement new strategies in their area of practice. It is recognised that there is a need for the development of a formal protocol for oral care assessment, for hospitalised patients. The absence of a protocol may influence nurses' practice and lead to missed care. Literature supports that some hospitals are very strict to such procedures and some others not and sometimes it depends on people's attitudes in each country in relation to oral care [38].

Another important facilitator that participants mentioned was nurses' continuing education on oral care which enhance their awareness and sensitiveness to this 
nursing procedure as Juan et al (2011) suggested [33]. In the current study most of the nurse's knowledge on oral care derived from their basic nursing training as in all countries, with no evidence of follow up in any continuous education program. It is also interesting that many times nurses are struggling to complete their work including mouthcare, but unfortunately they may underestimate the importance of patient education and training for example teaching a patient with cancer the necessity of careful mouthcare. There is evidence that patient education is considered a low priority in nursing intervention and is usually omitted, for example among patients in oncology and intensive care units $[39,40]$.

A factor that was strongly reported by the participants as crucial for the nurse's compliance on oral hygiene, was the role of the nurse manager and the control he/she performs in order to confirm that oral care is adequate and appropriate. However, although this factor was reported during the discussions, it isn't supported from the current literature.

\section{Conclusions}

Through this research it has been found that oral care is often omitted by nurses who work in the nursing departments and that there are many factors that contribute negatively or positively to its implementation. These factors focus on the patients' condition and their characteristics, the knowledge and perceptions of the nurses' and the working environment in general. Continuous education on oral care is vital for nurses in order to overcome the current perception that oral hygiene is less important that other tasks. Special training could also be offered to hospitalized patients as well, to assist help understand the necessity of oral care. This effort by all involved, may 
lead to improvement of the patients' health care status in order to minimize side effects during their hospitalization.

\section{Declarations}

\section{Ethics approval and consent to participate}

The research was carried out with the consent of the Cyprus National Bioethics Committee and was conducted in accordance with the Declaration of Helsinki ethical principles. To take part in this research, all study participants signed a voluntary written consent.

\section{Consent for Publication}

Not applicable

\section{Availability of data and materials}

All data generated or analysed during this study are included in this published article

\section{Affiliations}

Educational Sector, Nursing Services, Ministry of Health Cyprus Melanie Charalambous

Educational Sector, Nursing Services, Ministry of Health Cyprus Georgios Efstathiou

Dental Services, Ministry of Health

Persefoni Lambrou-Christodoulou

Educational Sector, Nursing Services, Ministry of Health Cyprus Iraklis Irakleous 
Department of Nursing, Vragadinou Street Limassol Cyprus University of Technology, Evridiki Papastavrou

\section{Corresponding author}

Correspondence to Melanie Charalambous

\section{Contributions}

EP and PL conceived and designed the study. II conducted the data collection and GE and MC transcription of the interviews. MC, GE and PL conducted the data analysis. EP, MC, GE, PL, II. interpreted the data. PL, MC, GE and II wrote several drafts of the manuscript. EP critically reviewed the manuscript. All authors have read, revised and approved the manuscript.

\section{Competing interests}

The authors declare that they have no competing interests.

\section{Acknowledgements}

The authors would like to thank all the nurse managers and nurses who took part in this study and provided their valuable experiences. In addition, they would like to thank Cyprus University of Technology for supporting open access publication.

\section{Funding}

Not applicable

\section{REFERENCES}

1. Jin LJ, Lamster IB, Greenspan JS, Pitts NB, Scully C, Warnakulasuriya S. Global burden of oral diseases: emerging concepts, management and interplay with systemic health. Oral Diseases. 2016; (7):609-19.

2. Abnet, CC, Qiao YL, Dawsey SM, Dong ZW, Taylor PR, Mark SD. 'Tooth loss is associated with increased risk of total death and death from upper 
gastrointestinal cancer, heart disease, and stroke in a Chinese populationbased cohort'. International Journal of Epidemiology. 2005; 34(2): 467-474.

3. Alhajj, MN, Halboub E, Amran A G, et al. Link between perceived oral and general health status among Yemeni adult dental patients. BMC Oral Health. BioMed Central, 2019; (1) 93:1-9.

4. Azarpazhooh A, Leake JL. Systematic Review of the Association between Respiratory Diseases and Oral Health. Journal of Periodontology. 2006; 77(9): 1465-1482.

5. Barbato L, Francioni E, Bianchi M, et al. Periodontitis and bone metabolism. Clinical Cases in Mineral and Bone Metabolism. 2015; 12 (2):174-177.

6. Terpenning MS, Taylor GW, Lopatin DE, et al. Aspiration Pneumonia: Dental and Oral Risk Factors in an Older Veteran Population, Journal of the American Geriatrics Society. 2001; 49(5), pp. 557-563.

7. Desvarieux M, Demmer RT, Rundek T, et al. Relationship between periodontal disease, tooth loss, and carotid artery plaque: the Oral Infections and Vascular Disease Epidemiology Study (INVEST).Stroke. 2003; 34(9), pp. 2120-5.

8. Jordão HW, McKenna G, McMenamin ÚC, et al. The association between selfreported poor oral health and gastrointestinal cancer risk in the UK Biobank: A large prospective cohort study. United European Gastroenterology Journal 2019; 7(9):1241-1249.

9. Gaiser R A, Halimi A, Alkharaan H, et al. Enrichment of oral microbiota in early cystic precursors to invasive pancreatic cancer. Gut. 2019; 0:1-9. 
10. Kalisch BJ, Landstrom, G. Williams, RA. Missed nursing care: Errors of omission. Nursing Outlook, 2009; 57(1):3-9.

11. Vryonides, S. Papastavrou E, Charalambous A, et al. The ethical dimension of nursing care rationing. A thematic synthesis of qualitative studies. Nursing Ethics. 2015; 22(8): 881-900.

12. Papastavrou E, Andreou P, Efstathiou G. Rationing of nursing care and nursepatient outcomes: A systematic review of quantitative studies. International Journal of Health Planning and Management. 2014: 29(1), 3-25.

13. Aiken LH, Clarke SP, Sloane DM, et al. Nurses' reports on hospital care in five countries. Health Affairs. 2001; 20:43-53.

14. Watson CA. Understanding the factors that influence nurses' job satisfaction. JONA. 2002; 32: 229-31.

15. Bowles C, Candela L. First job experiences of RN graduates: Improving the work environment. JONA 2005; 14:16-9.

16. Malkin B.The importance of patients' oral health and nurses' role in assessing and maintaining it. Nursing Times.2009; 105 (17):19-23.

17. Huskinson $\mathrm{W}$, Lloyd $\mathrm{H}$. Oral health in hospitalised patients: assessment and hygiene. Nursing Standard. 2009; 23 (36) 43-47.

18. Alja'afreh MA, Sultan MM, Habashneh SS. Nurses' perception and attitudes towards oral care practices for mechanically ventilated patients. Saudi Med J. 2018; Vol. 39 (4): 379-385.

19. Vrionides S, Papastavrou E, Charalambous A, et al. Ethical climate and missed nursing care in cancer care units. Nursing Ethics. 2018; 25(6): 707-723. 
20.Papastavrou E, Efstathiou G, Andreou C. Nursing students' perceptions of patients' dignity. Nursing Ethics. 2012; 23(1)92-103.

21.Zelenikova R, Drach-Zahavy A, Gurkova E, Papastavrou E. Understanding the concept of missed nursing care from a cross-cultural perspective. Journal of Advance Nursing. 2019; 75(11):2995-3005.

22. Krueker R, Casey MA. Focus Groups: A Practical Guide for Applied Research. 2015 Sage Publications $5^{\text {th }}$ edn.

23. Jones H, Newton J, Bower E. A Survey of the Oral Care Practices of Intensive Care Nurses. Intensive and Critical Care Nursing.2004; 20: 69-76.

24. Rachel K, Andrew B, Malcom B. Oral Hygiene Practices in Scottish Intensive Care Units: A National Survey the Intensive Care Society. Journal of the Intensive Care Society. 2009; 10:155-157.

25. Grap MJ, Munro C, Ashtiani B, Bryant S. Oral Care Interventions in Critical Care. American Journal of Critical Care. 2003; 12: 113-119.

26. Munro CL, Grap MJ, Kleinpell R. Oral health and care in the intensive care unit: state of the science. Am J Crit Care. 2004; 13: 25-34.

27. Ibrahim MS, Mudawi AM, Omer O. Nurses' Knowledge, Attitude and Practice of Oral Care for Intensive Care Unit Patients. Open Journal of Stomatology. 2015; 5: 179-186.

28. Alotaibi AK, Alotaibi SK, Alshayiqi M, Ramalingam S. Knowledge and attitudes of Saudi intensive care unit nurses regarding oral care delivery to mechanically ventilated patients with the effect of healthcare quality accreditation. Saudi $J$ Anaesth. 2016; 10(2):208-12. 
29. Pai RR, Ongole R. Nurses' knowledge and education about oral care of cancer patients undergoing chemotherapy and radiation therapy. Indian J Palliat Care. 2015; 21: 225-30.

30. Edward K, Mahoney AM, Felstead B, "Hospitalised mental health patients and oral health," Journal of Psychiatric Mental Health Nursing. 2012; 19: 419-425.

31. Ragotero IG, Balabagno AO, Rodriguez HM. Impact of oral health education program (OHEP) on competencies among nurses caring for totally dependent patients in two government tertiary hospitals in the Philippines. Philipp J Health Res Dev. 2015; $19: 6$.

32. de Lugt-Lustig KH, Vanobbergen JN, van der Putten GJ, et al. Effect of oral healthcare education on knowledge, attitude and skills of care home nurses: A systematic literature review. Community Dent Oral Epidemiol. 2014; 42:88-96.

33. Juan S, Helen P. Sonja, W. A Survey of Oral Care Practices in South African Intensive Care Units. SAJCC. 2011; 27, 42-46.

34. Salamone K, Yacoub E, Mahoney AM, Karen-leigh E. Oral Care of Hospitalised Older Patients in the Acute Medical Setting. Nursing Research and Practice. 2013; 2013:827670.

35. Papastavrou E, Andreou P, Efstathiou G. Rationing of nursing care and nursepatient outcomes: A systematic review of quantitative studies. Int J Health Plann Manage. 2014; 29(1):3-25.

36. Pai RR, Ongole R, Banerjee S. Oral care in cancer nursing: Practice and barriers. Indian Journal of Dental Research. 2019; 30 ( 2): 226-230.

37. Mukhtar A, Afzal M, Sarwar H, et al. Knowledge, Attitude and Practices of Nurses to Oral Care for Hospitalized Patients in Services Hospital, Lahore Saudi J. Med. Pharm. Sci. 2017; 3 (5):399-407. 
38. Butani Y, Weintraub JA, Barker J C. Oral health-related cultural beliefs for four racial/ethnic groups: Assessment of the literature. BMC Oral Health. 2008; 8: 26:1-13.

39. Papastavrou E, Vryonides S, Charalambous A and Eleftheriou C. To what extent are patients' needs met on oncology units? The phenomenon of care rationing European Journal of Oncology Nursing. 2016; 21:48-56.

40. O Labeau S, Van de Vyver K, Brusselaers N, Vogelaers D et al. Prevention of ventilator-associated pneumonia with oral antiseptics: a systematic review and meta-analysis. Lancet Infect Dis. 2011;11(11):845-54. 